Results About $52.6 \%$ in 2003 and $67.4 \%$ in 2008 of women aged 40 or older reported receipt of a mammogram. Compared to women 40-49 years old, those aged 50-69 had higher odds of having been screened (1.076 in 2003 and 1.354 in 2008), those aged 70 or older had lower odds (0.513 in 2003 and 0.625 in 2008). The odds increase with family income, education, being married, seeing a physician. Having insurance doubles the odds, as does living in a metropolitan area (3.620 in 2003 and 3.322 in 2008). Compared to the North region, residents in all other regions had larger odds.

Conclusions The age-group targeted by the national policy had marked increase in screening coverage. There are indications of a lessening in inequalities due to income levels, but disparities linked to regional variation have not been reduced.

\section{P1-270 SMALL AND LARGE FOR GESTATIONAL AGE CHILDREN HAVE DIFFERENT EATING BEHAVIOURS AT 6 MONTHS OF AGE}

doi:10.1136/jech.2011.142976e.62

\begin{abstract}
${ }^{1,2} \mathrm{~A}$ Oliveira, ${ }^{*} \mathrm{E}$ Pinto, ${ }^{3} \mathrm{P}$ Moreira, ${ }^{1,2} \mathrm{~A}$ C Santos, ${ }^{1,2} \mathrm{H}$ Barros, ${ }^{1,2} \mathrm{C}$ Lopes. ${ }^{1} \mathrm{Public}$ Health Institute of the University of Porto Medical School, Porto, Portugal; ${ }^{2}$ Department of Hygiene and Epidemiology of the University of Porto Medical School, Porto, Portugal; ${ }^{3}$ Faculty of Nutrition and Food Sciences of the University of Porto, Porto, Portugal
\end{abstract}

Aim To relate eating behaviours at 6 months of age with weight for gestational age at birth.

Methods Study subjects belong to a population-based birth cohort assembled in Porto, Portugal (Generation XXI, $n=8666$ ). A subcohort of 1562 newborns was re-evaluated at 6 months and 1227 singletons presented data on variables of interest. Data were gathered by trained interviewers. Mother answered a questionnaire on socio-demographic, clinical and behavioural characteristics. Small for gestational age (SGA) and large for gestational age (LGA) were defined as $<10$ th and $>90$ th percentile, respectively, of sex-specific Kramer growth charts. OR and $95 \%$ CIs were obtained from unconditional logistic regression, after adjustment for sex, mother's age, education and pre-pregnancy body mass index, maternal smoking during pregnancy, and breastfeeding.

Results Approximately $15 \%$ of children were SGA and 4\% were LGA. Compared to adequate for gestational age children, SGA had more frequently mothers reporting difficulties in feeding at 6 months (OR $=1.52,95 \%$ CI 1.01 to 2.31$)$ and eating small quantities each time (OR=1.78, 95\% CI 1.27 to 2.49). LGA children had also more feeding difficulties ( $O R=2.26,95 \%$ CI 1.10 to 4.63$)$ and a higher probability of refusing solid foods $(\mathrm{OR}=2.21,95 \% \mathrm{CI} 1.02$ to 4.80). No associations were found neither with eating slowly, being angry at the meals' end, choke with food and spitting up milk, nor a later weaning or inclusion of fruits and vegetables as first foods.

Conclusions Both SGA and LGA children presented more feeding difficulties at 6 months of age, and LGA was associated with neophobia to solid foods.

\section{P1-271 PREVALENCE, RISK FACTORS AND PATTERNS OF CHRONIC KIDNEY DISEASE IN A RURAL COMIMUNITY IN SOUTH WEST NIGERIA}

doi:10.1136/jech.2011.142976e.63

${ }^{1} \mathrm{R}$ Oluyombo, ${ }^{*} \mathrm{~A}$ Akinsola, ${ }^{1} 0$ Ayodele, ${ }^{2} \mathrm{~A}$ Onayade, ${ }^{2} \mathrm{~F}$ Arogundade, ${ }^{2} \mathrm{~A}$ Sanusi, ${ }^{1} \mathrm{P}$ Akinwusi, ${ }^{1} 0$ Okunola. 'Ladoke Akintola University of Technology Teaching Hospital, Osogbo, Osun state, Nigeria; ${ }^{2}$ Obafemi Awolowo University Teaching Hospital, Ile-lfe, Osun state, Nigeria

Introduction Chronic kidney disease (CKD) is a global public health problem. Despite the long term difficulties of this condition there is paucity of community derived data in sub-Saharan Africa and especially in Nigeria, the most populated country in Africa. This lack of data is hampering an appropriate response.
Methods Adults (aged $\geq 18$ years) were randomly selected. A structured questionnaire was used to collect data on sociodemographic characteristics and knowledge of kidney disease. Clinical examination was undertaken including: anthropometry, blood pressure, fasting or random blood sugar, dipstick urinalysis, albumin to creatinine ratio and urine microscopy. Glomerular filtration rate (GFR) was estimated using the Modification of Diet in Renal Disease (MDRD) equation.

Results The mean age of participants was $45.8 \pm 19.0$ years with a male: female ratio 0.8:1. 19\% consumed alcohol and 7\% smoked. $20 \%$ used regular analgesia and $75 \%$ used herbal concoctions. The prevalence of hypertension was $30 \%$, diabetes mellitus (DM) (3.7\%), obesity (defined by BMI) $2.7 \%$ and elevated waist circumference (14.6\%). Urine microscopy revealed: haematuria $3.1 \%$, ova of Schistosoma haematobium $1.1 \%$ and macroalbuminuria (8.9\%). An estimated GFR $<60 \mathrm{ml} / \mathrm{min} / 1.73 \mathrm{~m}^{2}$ occured in $12.3 \%$. The prevalence of CKD was $18.8 \%$. Increasing age (OR 0.92, 95\% CI 0.88 to 0.96 ), female gender (OR 4.87, 95\% CI 1.34 to 17.74 ), systolic blood pressure (OR 1.04, 95\% CI 1.01 to 1.07) and DM (OR 15.76, 95\% CI 1.25 to 199.24$)$ were predictive of CKD.

Conclusion $\mathrm{CKD}$ and its risk factors are prevalent in this community. The majority had moderately impaired kidney function. There is need for both primary and secondary preventive programmes.

\section{P1-272 EVALUATION OF EFFECTS OF ATOMIC BOMB SURVIVORS' HEALTH HANDBOOKS ON THEIR HEALTH PROMOTION}

doi:10.1136/jech.2011.142976e.64

K Otani, ${ }^{*}$ M Ohtaki, K Satoh, T Tonda. Hiroshima University, Hiroshima, Japan

Introduction Atomic bombs were dropped on Hiroshima and Nagasaki in 1945, and then A-bomb survivors' health handbooks (shortly 'handbooks') were issued by the Japanese government to help Abomb survivors in 1957. They have been able to receive free medical checkup twice for a year and free medical care for designated disorders. The purpose of the study is to evaluate effects of A-bomb survivors' health handbooks focusing on the relationship between a mortality risk and a length of having handbook.

Methods Objects for analysis were selected from the ABS database of RIRBM Hiroshima University. The number of over-all deaths is 58599 and the number of censored data is 101244 . Cox's proportional hazard model was applied for analysing the data. The observation period is from 1970 to 1997 and the time variable is a time from $1^{\text {st }}$ January 1970 to an occurrence of death. Length of having a handbook was defined as the period from registration year as an A-bomb survivor to 1970. Sex, age at A-bomb exposure, radiation dose and a length of having a handbook are used as covariates.

Results and Conclusion In men, there was significant negative relationship between a length of having a handbook and a relative mortality risk after being adjusted for sex, radiation dose and age at bombing, but in women such a relationship was not found. A man who got a handbook at a young age had a lower mortality risk compared to a man at an old age.

\section{P1-273 THE CHANGING ROLE OF MORTALITY PREDICTORS OVER 20 YEARS OF OBSERVATION}

doi:10.1136/jech.2011.142976e.65

A Pac, ${ }^{*}$ B Tobiasz-Adamczyk, P Brzyski, M Brzyska, M Florek. Jagiellonian University Medical College, Chair of Epidemiology and Preventive Medicine, Krakow, Poland

Longitudinal studies among elderly are concentrated on finding the predictors of mortality. Still, there is an open question if those 